\title{
On the extension of the Namioka-Klee theorem and on the Fatou property for Risk Measures
}

\author{
Sara Biagini, Marco Frittelli \\ In: Optimality and risk: modern trends in mathematical \\ finance. The Kabanov Festschrift (2009) \\ Editors: F. Delbaen, M. Rasonyi, Ch. Stricker
}

\begin{abstract}
This paper has been motivated by general considerations on the topic of Risk Measures, which essentially are convex monotone maps defined on spaces of random variables, possibly with the so-called Fatou property.

We show first that the celebrated Namioka-Klee theorem for linear, positive functionals holds also for convex monotone maps $\pi$ on Frechet lattices.

It is well-known among the specialists that the Fatou property for risk measures on $L^{\infty}$ enables a simplified dual representation, via probability measures only. The Fatou property in a general framework of lattices is nothing but the lower order semicontinuity property for $\pi$. Our second goal is thus to prove that a similar simplified dual representation holds also for order lower semicontinuous, convex and monotone functionals $\pi$ defined on more general spaces $\mathcal{X}$ (locally convex Frechet lattices). To this end, we identify a link between the topology and the order structure in $\mathcal{X}$ - the C-property - that enables the simplified representation. One main application of these results leads to the study of convex risk measures defined on Orlicz spaces and of their dual representation.
\end{abstract}

Acknowledgements The first author would like to thank B. Rudloff, P. Cheridito and A. Hamel for some discussions while she was visiting the ORFE Department at Princeton University. The second author would like to thank Marco Maggis, PhD student at Milano University for helpful discussion on this subject.

\section{Introduction}

The analysis in this paper was triggered by recent developments in the theory of Risk Measures in Mathematical Finance. Convex risk measures were 
independently introduced by [FS02] and [FR02] as generalization of the concept of a coherent risk measure developed in [ADEH99]. Consider a space of financial positions $\mathcal{X}$ (real-valued, measurable functions on a fixed measurable space $(\Omega, \mathcal{F})$ ) containing the constants. A convex risk measure on $\mathcal{X}$ is a map $\rho: \mathcal{X} \rightarrow(-\infty,+\infty]$ with the following properties:

1. $\rho(0)=0$ (so $\rho$ is proper, i.e. it does not coincide with $+\infty$ )

2. monotonocity: if $X, Y \in \mathcal{X}, X \leq Y$, then $\rho(X) \geq \rho(Y)$

3. convexity: if $\lambda \in[0,1]$, then $\rho(\lambda X+(1-\lambda) Y) \leq \lambda \rho(X)+(1-\lambda) \rho(Y)$ for any $X, Y \in \mathcal{X}$

4. cash additivity: if $m \in \mathbb{R}$ then $\rho(X+m)=\rho(X)-m$ for any $X \in \mathcal{X}$

When $\rho$ is also positive homogeneous, i.e.

5. $\rho(\lambda X)=\lambda \rho(X)$ for all $\lambda \geq 0$

the risk measure is called coherent.

If $\mathcal{X}$ is also topological space (as it is always the case in the applications), it is of course useful to have a result on the degree of smoothness of the risk measure $\rho$. Strangely enough, when this paper was first written to our knowledge there was yet no general result. This is exactly the message of the extended NamiokaKlee Theorem, stated below in Theorem 1. The (topological vector) space of positions $\mathcal{X}$ however must have some other properties, i.e. it must be a Frechet lattice.

Recall that a topological vector space $(\mathcal{X}, \tau)$ is a Frechet lattice if:

- its topology $\tau$ is induced by a complete distance $d$

- $\mathcal{X}$ is a lattice, that is it has an order structure $(\mathcal{X}, \leq)$ and each pair $X_{1}, X_{2} \in \mathcal{X}$ has a supremum $X_{1} \vee X_{2}$ in $\mathcal{X}$

- $\mathcal{X}$ is locally solid, that is the origin 0 has a fundamental system of solid neighborhoods (a neighborhood $U$ of 0 is solid if for any $X \in U, Y \in$ $\mathcal{X},|Y| \leq|X| \Rightarrow Y \in U$ where $|X|=X \vee(-X))$.

Note that a Frechet lattice is not necessarily locally convex. Examples of common Frechet lattices are the spaces $L^{p}$ on a probability space $(\Omega, \mathcal{F}, P)$, for $p \in[0,1)$ (with the natural, a.s. pointwise order). When $p \geq 1, L^{p}$ is also Frechet lattice, but with an extra property. The topology is induced by the $L^{p}$-norm (and thus the space becomes locally convex). Moreover, the norm has a monotonicity property: $|X| \leq|Y| \Rightarrow\|X\|_{p} \leq\|Y\|_{p}$. So $L^{p}, p \in[1,+\infty]$ is in 
fact a Banach lattice. Other Banach lattices important for our applications belong to the family of Orlicz spaces - denoted with $L^{\Psi}$ for a Young function $\Psi$ - which are described in details in Section 5.

Finally, we present the abstract statement of the extended Namioka-Klee Theorem, proved in Section 2 (where there is also an extensive comparison with the existing literature, as we discovered that there are a couple of recent, very similar results). The Theorem is stated for convex, monotone increasing maps $\pi$, not necessarily cash additive. But a similar result clearly holds for monotone decreasing maps. $\operatorname{Dom}(\pi)$ indicates here and in the rest of the paper the subset of $\mathcal{X}$ where $\pi$ is finite. The topological dual space is denoted by $\mathcal{X}^{\prime}$ and the set $\mathcal{X}_{+}^{\prime}$ indicates the convex cone of those functionals $Y$ in $\mathcal{X}^{\prime}$ that are positive, i.e. $\langle Y, X\rangle \geq 0$ for all $X \in \mathcal{X}, X \geq 0$. The symbol $\langle$,$\rangle indicates the bilinear form$ for the duality $\left(\mathcal{X}, \mathcal{X}^{\prime}\right)$. The map $\pi^{*}: \mathcal{X}^{\prime} \rightarrow(-\infty,+\infty]$ is the convex conjugate of $\pi$, also known as Fenchel transform, and it is defined as

$$
\pi^{*}(Y)=\sup _{X \in \mathcal{X}}\{\langle Y, X\rangle-\pi(X)\} .
$$

Theorem 1 (Extended Namioka-Klee) Any proper convex and monotone increasing functional $\pi: \mathcal{X} \rightarrow(-\infty,+\infty]$ on a Frechet lattice $(\mathcal{X}, \tau)$ is continuous and subdifferentiable on $\operatorname{int}(\operatorname{Dom}(\pi)$ ) (the interior of Dom $(\pi)$ ). Moreover, it admits a dual representation as

$$
\pi(X)=\max _{Y \in \mathcal{X}_{+}^{\prime}}\left\{\langle Y, X\rangle-\pi^{*}(Y)\right\} \quad \forall X \in \operatorname{int}(\operatorname{Dom}(\pi)) .
$$

To give an idea about the genesis of the second and most innovative part of the paper, let us go back to the financial setup and let us focus first on the case $L^{\infty}$. A risk measure $\rho$ on $L^{\infty}$ has the pleasant property of being always finitevalued, thanks to the boundedness of its elements and to the monotonicity and cash additivity property. The theorem above ensures that $\rho$ is continuous and subdifferentiable on the entire $L^{\infty}$. This implies the existence of a well-known dual representation for $\rho$ over $L^{\infty}$, namely

$$
\rho(X)=\max _{Q \in \mathcal{M}_{1, f}(P)}\left\{E_{Q}[-X]-\rho^{*}(Q)\right\}
$$

where:

a) $\mathcal{M}_{1, f}(P)$ indicates the set of positive, finitely additive measures $Q$ on $(\Omega, \mathcal{F})$ that are absolutely continuous w.r. to $P$ and are normalized $\left(Q\left(I_{\Omega}\right)=1\right)$;

b) $\rho^{*}$ is the convex conjugate of $\rho$ and should be interpreted as a penalty functional.

These results are known and proved e.g. [FS04, Theorem 4.12]), where subdifferentiability is proved by hand for the specific case study $L^{\infty}$.

Now, let us recall the definition of the Fatou property for risk measures (see e.g. [De00] or Section 4, [FS04], in the case $L^{\infty}$ ): 
Definition 2 (Fatou property) A risk measure $\rho: L^{p} \rightarrow \mathbb{R} \cup\{\infty\}, p \in$ $[1, \infty]$, has the Fatou property (F.P.) if given any sequence $\left\{X_{n}\right\}_{n}$ dominated in $L^{p}$ and converging $P-$ a.s. to $X$ we have:

$$
\rho(X) \leq \liminf _{n \rightarrow \infty} \rho\left(X_{n}\right)
$$

This property enables a simplified dual representation of $\rho$. Instead of the finitely additive measures $\mathcal{M}_{1, f}(P)$, if a convex risk measure $\rho: L^{\infty} \rightarrow \mathbb{R}$ has the F.P. one can write

$$
\rho(X)=\sup _{Q \text { probability, } Q \ll P}\left\{E_{Q}[-X]-\rho^{*}(Q)\right\},
$$

so the supremum can be taken only over probabilities, the $\sigma$-additive elements of $\mathcal{M}_{1, f}$, see [FS04, Theorem 4.26]. There is a price to pay: the above supremum may not be attained over probability measures, but only on $\mathcal{M}_{1, f}$.

What can be said about the representation problem of a convex risk measures defined on subspaces of $L^{0}$ ?

The spaces $L^{p}$ are typical examples of spaces of financial positions in the applications (see for example, [FR02], [FS07], [RK08]). Moreover, in [BF08] it is shown that Orlicz spaces that can be associated with a utility function are the right framework for the utility maximization problems which commonly arises in financial problems. Motivated by this idea, in the first version of this paper ${ }^{1}$, we initiated the study of risk measures defined on Orlicz spaces $L^{\Psi}$ and more generally on Frechet lattices. Independently, in [CL07] convex risk measures were defined on the Morse subspace $M^{\Psi}$ of the Orlicz space $L^{\Psi}$. As we shall see in Section 5 some of the findings in [CL07] are special case our results, while other properties do not hold in the general case, essentially because the topology on the (whole) Orlicz space $L^{\Psi}$ is not order continuous.

Our generalization of the representation in (3) and its various implications will be stated for maps $\pi$, defined of general Frechet lattice, that are convex, monotone and increasing, not necessarily translation invariant. This latter implies that the set of dual variables over which the supremum - or the maximum - is taken will not be normalized in general.

To begin with, let us recall a few notions about Riesz spaces, i.e. linear spaces that are lattices (see also Section 3). The first is that of order convergence. A generalized sequence, or net, $\left(X_{\alpha}\right)_{\alpha}$ in a Riesz space $\mathcal{R}$ is order convergent to some $X \in \mathcal{R}$, notation $X_{\alpha} \stackrel{o}{\rightarrow} X$, if there is a net $\left(Z_{\alpha}\right)_{\alpha}$ in $\mathcal{R}$ satisfying

$$
Z_{\alpha} \downarrow 0 \text { and }\left|X_{\alpha}-X\right| \leq Z_{\alpha} \text { for each } \alpha
$$

\footnotetext{
${ }^{1}$ Presented by the second author at the Workshop on Risk Measures, University of Evry, France, July 6-7 2006 and at the Conference on Risk Measures and Robust Control in Finance, The Bendheim Center, Princeton University, October 6-7, 2006.
} 
( $Z_{\alpha} \downarrow 0$ means that $\left(Z_{\alpha}\right)_{\alpha}$ is monotone decreasing and its infimum is 0 ).

A functional $f: \mathcal{R} \rightarrow \mathbb{R}$ defined on $\mathcal{R}$ is order continuous if

$$
X_{\alpha} \stackrel{o}{\rightarrow} X \Rightarrow f\left(X_{\alpha}\right) \rightarrow f(X),
$$

and a topology $\tau$ on $\mathcal{R}$ is order continuous if

$$
X_{\alpha} \stackrel{o}{\rightarrow} 0 \Rightarrow X_{\alpha} \stackrel{\tau}{\rightarrow} 0 .
$$

These definitions readily imply that if the topology $\tau$ is order continuous then

$$
f \text { is (topologically) continuous } \Rightarrow f \text { is order continuous. }
$$

We denote with $\left(\mathcal{X}_{n}^{\sim}\right)$ the cone of order continuous linear functionals on $\mathcal{X}$. By the classic Namioka Theorem, see Section 2., $\left(\mathcal{X}_{n}^{\sim}\right)_{+}$coincides with $\mathcal{X}_{+}^{\prime}$, the positive elements of topological dual space $\mathcal{X}^{\prime}$. We recall that in a Banach lattice $\mathcal{X}$ the norm topology is order continuous if and only if $\mathcal{X}_{n}^{\sim}=\mathcal{X}^{\prime}$ and that the following three classes of spaces all have order continuous norm: (a) $L^{p}$ when $p \in[1,+\infty)$; (b) $M^{\Psi}$ when $\Psi$ is a finite valued Young function; (c) $L^{\Psi}$ when $\Psi$ is a Young function satisfying the $\Delta_{2}$ condition (in this case $L^{\Psi}=M^{\Psi}$ ).

The above implication, together with Theorem 1, readily imply the following

Corollary 3 If Frechet lattice $(\mathcal{X}, \tau)$ has an order continuous topology and $\pi$ : $\mathcal{X} \rightarrow \mathbb{R}$ is convex and monotone (increasing), $\pi$ is already order continuous on $\mathcal{X}$. Thus it admits a dual representation as

$$
\pi(X)=\max _{Y \in\left(\mathcal{X}_{\tilde{n}}^{\tilde{n}}\right)_{+}}\left\{\langle Y, X\rangle-\pi^{*}(Y)\right\}, \quad X \in \mathcal{X},
$$

where $\left(\mathcal{X}_{n}^{\sim}\right)_{+}=\mathcal{X}_{+}^{\prime}$.

a) In the specific case $\mathcal{X}=L^{p}, p \in[1,+\infty)$ the representation above becomes

$$
\pi(X)=\max _{Y \in\left(L^{q}\right)_{+}}\left\{E[Y X]-\pi^{*}(Y)\right\}, \quad X \in L^{p},
$$

b) In the specific case $\mathcal{X}=M^{\Psi}$ and $\Psi$ is a finite valued Young function, the representation above becomes

$$
\pi(X)=\max _{Y \in\left(L^{\Psi^{*}}\right)_{+}}\left\{E[Y X]-\pi^{*}(Y)\right\}, \quad X \in M^{\Psi},
$$

where $\Psi^{*}$ is the conjugate function of $\Psi$.

However, the order continuity of a topology is a strong assumption, which is not satisfied by e.g. $L^{\infty}$, or by $L^{\Psi}$ for general Young functions $\Psi$ or by other 
Frechet lattices, as shown in Section 5. Moreover, in general $\left(\mathcal{X}_{n}^{\sim}\right)_{+}$is only a subspace of $\mathcal{X}_{+}^{\prime}$. This is exactly what happens with $L^{\infty}$ :

$$
\left(\left(L^{\infty}\right)_{n}^{\sim}\right)_{+}=L_{+}^{1} \text { and }\left(L^{\infty}\right)_{+}^{\prime}=L_{+}^{1} \oplus S_{+},
$$

where $S$ are the purely finitely additive measures.

It is then natural to investigate whether $\pi$ admits a representation on $\left(\mathcal{X}_{n}^{\sim}\right)_{+}$ under conditions, linking topology and order structure, less restrictive than the order continuity of the topology $\tau$.

As we shall see in Remark 17, when $\mathcal{X}=L^{p}, p \in[0, \infty]$, the Fatou property coincides with order lower semicontinuity, which is the appropriate concept in the present general setting.

Definition 4 A functional $\pi: \mathcal{R} \rightarrow(-\infty,+\infty]$ defined on a Riesz space $\mathcal{R}$ is order lower semicontinuous if $X_{\alpha} \stackrel{o}{\rightarrow} X$ implies $\pi(X) \leq \liminf \pi\left(X_{\alpha}\right)$.

From now on, local convexity is needed and in what follows the Frechet lattice $\mathcal{X}$ is also supposed locally convex.

As a consequence of the Hahn-Banach theorem in any locally convex Frechet lattice if the proper, increasing convex map $\pi: \mathcal{X} \rightarrow(-\infty,+\infty]$ is also $\sigma\left(\mathcal{X}, \mathcal{X}_{n}^{\sim}\right)$ lower semicontinuous then

$$
\pi(X)=\sup _{Y \in\left(\mathcal{X}_{\tilde{n}}^{\sim}\right)_{+}}\left\{\langle Y, X\rangle-\pi^{*}(Y)\right\}, \quad X \in \mathcal{X},
$$

where $\pi^{*}$ is the convex conjugate of $\pi$. Therefore $\pi$, as the pointwise supremum of a family of order continuous functionals, is also order lower semicontinuous

One could then conjecture that the converse always holds true i.e. any order lower semicontinuous $\pi$ on any locally convex Frechet lattice admits a representation in terms of $\left(\mathcal{X}_{n}^{\sim}\right)_{+}$, as in (7) or, in lucky cases, (5).

The conjecture is not true in general, see Example 6 at the end of this Introduction. Proposition 24 contains the main result of the paper, that is there exists an additional assumption "à la Komloś", linking the topology $\tau$ and the order structure, that enables the representation over $\left(\mathcal{X}_{n}^{\sim}\right)_{+}$.

Definition 5 (C-property) A linear topology $\tau$ on a Riesz space has the $C$ property ${ }^{2}$ if $X_{\alpha} \stackrel{\tau}{\rightarrow} X$ implies the existence of a subsequence $\left(X_{\alpha_{n}}\right)_{n}$ and convex combinations $Z_{n} \in \operatorname{conv}\left(X_{\alpha_{n}}, \cdots\right)$ such that $Z_{n} \stackrel{\circ}{\rightarrow} X$.

This property is quite reasonable, all the details are in Section 4. In particular, whenever a locally convex Frechet lattice $(\mathcal{X}, \tau)$ can be embedded in $L^{1}$ with a linear lattice embedding, then all the topologies: $\tau, \sigma\left(\mathcal{X}, \mathcal{X}^{\prime}\right)$ and $\sigma\left(\mathcal{X}, \mathcal{X}_{n}^{\sim}\right)$

\footnotetext{
${ }^{2}$ The "C" stands for "convex combinations"...
} 
have the C-property. A relevant example of spaces with an associated collection of topologies (norm, weak and $\sigma\left(\mathcal{X}, \mathcal{X}_{n}^{\sim}\right)$ ) satisfying the C-property is the family of Orlicz spaces (Section 5.1).

In the case $\mathcal{X}=L^{\infty}$, it is well known that the sup in (7) in general is not a max, but the sup is attained under some stronger continuity condition. In the general case, in Lemma 27 we show that for a finite valued convex increasing map which is order upper semicontinuous the sup in (7) is indeed a max.

Finally, in Section 5.2 we analyze convex risk measures defined on Orlicz spaces and with values in $\mathbb{R} \cup\{+\infty\}$. This new setup allows for an extension of the known dual representation on $L^{\infty}$. We further provide some new results on the convex risk measures associated to utility functions, as in the case of the entropic risk measure.

Example 6 (When the C-property fails) When the $\sigma\left(\mathcal{X}, \mathcal{X}_{n}^{\sim}\right)$ - topology does not satisfy the C-property, there may be order l.s.c. convex functionals (even $\tau$ continuous!) that are not $\sigma\left(\mathcal{X}, \mathcal{X}_{n}^{\sim}\right)-$ l.s.c.

Take $\mathcal{X}=C([0,1])$, the Banach lattice of the continuous functions on $[0,1]$ with the supremum norm and the pointwise order. The dual $\mathcal{X}^{\prime}$ consists of the Borel signed measures on $[0,1]$ and it is known (see e.g. [Za83, Example $87.5])$ that there is no non zero order-continuous functional in $\mathcal{X}^{\prime}$. The topology $\sigma\left(\mathcal{X}, \mathcal{X}_{n}^{\sim}\right)=\sigma(\mathcal{X},\{0\})$ is therefore the indiscrete one and clearly it doesn't have the $C$-property.

Consider then the convex, increasing 'best case' functional

$$
\pi(X)=\max _{t \in[0,1]} X(t)
$$

which is finite valued, so that the extended Namioka-Klee Theorem implies that $\pi$ is norm continuous and subdifferentiable and it admits a representation on $\mathcal{X}_{+}^{\prime}$ as

$$
\pi(X)=\max _{Y \in \mathcal{X}_{+}^{\prime}}\left\{\langle Y, X\rangle-\pi^{*}(Y)\right\}
$$

But it evidently does not admit a representation on $\left(\mathcal{X}_{n}^{\sim}\right)_{+}=\{0\}$ because it is not constant.

To show that $\pi$ is order-l.s.c. let $X_{\alpha} \stackrel{o}{\rightarrow} X$ and suppose by contradiction that there exists a subnet $\left(X_{\alpha_{\beta}}\right)_{\beta}$ (as a subnet, still order convergent to $X$ ) such that $\pi(X)>\lim _{\beta} \pi\left(X_{\alpha_{\beta}}\right)$. Let $t^{*} \in \operatorname{argmax}(X)$. Then

$$
\pi\left(X_{\alpha_{\beta}}\right) \geq X_{\alpha_{\beta}}\left(t^{*}\right)
$$

and evidently

$$
X\left(t^{*}\right)=\pi(X)>\lim _{\beta} \pi\left(X_{\alpha_{\beta}}\right) \geq \limsup _{\beta} X_{\alpha_{\beta}}\left(t^{*}\right),
$$


which contradicts pointwise convergence.

\section{The Extended Namioka Theorem}

The following is the statement of the well-known Namioka-Klee Theorem in the case of linear functionals $\varphi$.

Theorem 7 (Namioka-Klee) Any linear and positive functional $\varphi: \mathcal{X} \rightarrow \mathbb{R}$ on a Frechet lattice $\mathcal{X}$ is continuous (see [Na57]).

In order to provide a technically straightforward, but quite relevant, extension of Namioka-Klee Theorem to convex functionals $\pi$, the positivity assumption

$$
0 \leq Y \Rightarrow 0 \leq \varphi(Y)
$$

has to be replaced with the requirement that $\pi$ is monotone increasing

$$
X \leq Y \Rightarrow \pi(X) \leq \pi(Y)
$$

Monotonicity and positivity are equivalent for linear functionals, but it is straightforward to see that for convex $\pi$ monotonicity implies positivity (assuming $\pi(0)=0)$. And it is easy to produce a positive but non-monotone convex map by taking $\pi(X)=|X|$ on $\mathcal{X}=\mathbb{R}$.

So, while on one hand one relaxes the linearity assumption, on the other hand a stronger link with the order structure is required.

The properties in the next Lemma are straightforward consequences of the definitions.

Lemma 8 Let $\mathcal{R}$ be a Riesz space and let $\pi: \mathcal{R} \rightarrow(-\infty,+\infty]$ be convex, increasing and $\pi(0)=0$. Then:

$$
\begin{aligned}
& i-\pi(\alpha X) \leq \alpha \pi(X), \forall \alpha \in[0,1], \forall X \in \mathcal{R} ; \\
& i i-\alpha \pi(X) \leq \pi(\alpha X), \forall \alpha \in(-\infty, 0] \cup[1+\infty), \forall X \in \mathcal{R} ; \\
& i i i-|\pi(X)| \leq \pi(|X|) \forall X \in \mathcal{R} .
\end{aligned}
$$

Proof of Theorem 1. Step 1: Continuity. The proof of Namioka-Klee Theorem (see e.g. [ABo05, Theorem 9.6]) can be adapted, in a straightforward manner, to deal with the current weaker assumptions. We repeat the argument so that the paper is self- contained.

W.l.o.g. it can be assumed that the interior of $\operatorname{Dom}(\pi)$, $\operatorname{int}(\operatorname{Dom}(\pi))$, is not empty that $0 \in \operatorname{int}(\operatorname{Dom}(\pi))$ and $\pi(0)=0$. Let $B_{r}$ be the centered open ball of radius $r>0$ in a metric that generates $\tau$. Take any sequence $\left\{X_{n}\right\}_{n}$ such that $X_{n} \stackrel{\tau}{\rightarrow} 0$. Fix $r$ small enough, so that $B_{2 r} \subseteq \operatorname{int}(\operatorname{Dom}(\pi))$. Then pick a 
countable base $\left\{V_{n}\right\}_{n}$ of solid neighborhoods of zero satisfying $V_{1}+V_{1} \subseteq B_{r}$ and $V_{n+1}+V_{n+1} \subset V_{n}$ for each $n$. Then $V_{n+1} \subseteq V_{n} \subseteq B_{r}$ for each $n$. By passing to a subsequence of $X_{n}$, one can suppose $X_{n} \in \frac{1}{n} V_{n}$ for each $n$. Set $Y_{n}=\sum_{i=1}^{n} i\left|X_{i}\right|$ and note that $Y_{n} \leq Y_{n+1}$ and $n\left|X_{n}\right| \leq Y_{n}$. In addition

$$
Y_{n+p}-Y_{n}=\sum_{i=n+1}^{n+p} i\left|X_{i}\right| \in V_{n+1}+V_{n+2}+\ldots+V_{n+p} \subset V_{n}
$$

Therefore $Y_{n} \in B_{r}$ for each $n$ and $\left\{Y_{n}\right\}_{n}$ is a Cauchy sequence, so $Y_{n} \stackrel{\tau}{\rightarrow} Y$ for some $Y$ in $\mathcal{X}$. Since $Y_{n} \in B_{r}, Y \in \overline{B_{r}} \subset B_{2 r} \subseteq \operatorname{int}(\operatorname{Dom}(\pi)), \pi(Y)$ is finite. This $Y$ is an upper bound for the sequence (actually, $\sup _{n} Y_{n}=Y$ ). In fact, fix any $n$. Since $Y_{m}-Y_{n} \in \mathcal{X}_{+}$for each $m \geq n$, the sequence $\left\{Y_{m}-Y_{n}: m \geq n\right\}$ in $\mathcal{X}_{+}$satisfies

$$
Y_{m}-Y_{n} \stackrel{\tau}{\rightarrow} Y-Y_{n}, \text { as } m \rightarrow \infty .
$$

Since $\mathcal{X}_{+}$is $\tau$-closed ([ABo05, Theorem $\left.8.43-1\right]$ ), $Y-Y_{n} \in \mathcal{X}_{+}$for each $n$. Hence $Y_{n} \leq Y$ for each $n$. From Lemma $8,\left|\pi\left(X_{n}\right)\right| \leq \pi\left(\left|X_{n}\right|\right) \leq \frac{1}{n} \pi\left(n\left|X_{n}\right|\right)$. By monotonicity of $\pi$ we derive

$$
\left|\pi\left(X_{n}\right)\right| \leq \frac{1}{n} \pi\left(n\left|X_{n}\right|\right) \leq \frac{1}{n} \pi\left(Y_{n}\right) \leq \frac{1}{n} \pi(Y) \rightarrow 0
$$

which shows that $\pi$ is continuous at zero and therefore $\pi$ is continuous on the whole $\operatorname{int}(\operatorname{Dom}(\pi))([\mathrm{ABo05}$, Theorem 5.43]).

Step 2: Subdifferentiability. For all $X^{*} \in \operatorname{int}(\operatorname{Dom}(\pi))$ we must exhibit a subgradient $Y^{*} \in \mathcal{X}^{\prime}$, i.e. a $Y^{*}$ such that

$$
\pi(X)-\pi\left(X^{*}\right) \geq\left\langle Y^{*}, X-X^{*}\right\rangle \text { for all } X \in \mathcal{X} .
$$

To this end, again w.l.o.g. we can suppose $X^{*}=0 \in \operatorname{int}(\operatorname{Dom}(\pi))$ and $\pi(0)=0$. Then, the directional derivative functional $D$ in 0

$$
D(X):=\lim _{t \downarrow 0} \frac{\pi(t X)}{t}
$$

satisfies $D \leq \pi$ thanks to Lemma 8 . It is finite valued and convex and thus the first part of this proof implies that it is continuous. By the Hahn-Banach Theorem (see e.g. [ABo05, Theorem 5.53]) there exists a linear functional $Y^{*}$ which satisfies $\left\langle Y^{*}, X\right\rangle \leq D(X)$ on $\mathcal{X}$ whence $Y^{*}$ is a continuous subgradient for $\pi$ at 0 .

Step 3: Representation. Fix any $X^{*} \in \operatorname{int}(\operatorname{Dom}(\pi))$. It is an exercise to show that $\pi$ increasing implies $\pi^{*}$ is finite at most over $\mathcal{X}_{+}^{\prime}$. Fix any subgradient $Y^{*}$ (which is then positive) of $\pi$ at $X^{*}$. Reshuffling eq. (8), this means

$$
\left\langle Y^{*}, X^{*}\right\rangle-\pi\left(X^{*}\right)=\max _{X \in \mathcal{X}}\left\{\left\langle Y^{*}, X\right\rangle-\pi(X)\right\}=\pi^{*}\left(Y^{*}\right)
$$


where the last equality follows from the definition of $\pi^{*}$. This chain of equalities in turn implies that $\pi\left(X^{*}\right)=\left\langle Y^{*}, X^{*}\right\rangle-\pi^{*}\left(Y^{*}\right)=\max _{Y \in \mathcal{X}^{\prime}}\left\{\left\langle Y, X^{*}\right\rangle-\pi^{*}(Y)\right\}$ as the inequality $\pi\left(X^{*}\right) \geq\left\langle Y, X^{*}\right\rangle-\pi^{*}(Y)$ automatically holds for any $Y \in \mathcal{X}^{\prime}$.

Remark 9 In [CLO7] there is a formula identical to (1) for $\pi$ defined on Banach lattices.

Corollary 10 Every finite-valued convex and monotone functional on a Banach lattice is norm-continuous and subdifferentiable.

Corollary 11 If a Frechet lattice $\mathcal{X}$ supports a non-constant convex monotone map $\pi$, then necessarily $\mathcal{X}^{\prime} \neq\{0\}$.

As a generic Frechet lattice $\mathcal{X}$ is not necessarily locally convex, it may happen that the topological dual $\mathcal{X}^{\prime}$ is very poor or even $\{0\}$. This is the case, for example, of the spaces $L^{p}(\Omega, \mathcal{F}, \mu), p \in(0,1)$, when $\mu$ is a nonatomic measure (see $\left[\mathrm{ABo} 05\right.$, Theorem 13.31]) and of the space $L^{0}(\Omega, \mathcal{F}, \mu)$, when $\mu$ is a nonatomic finite measure ( $[\mathrm{ABo} 05$, Theorem 13.41] ). Therefore, the only convex monotone $\pi \mathrm{s}$ on these spaces are the constants.

\subsection{The current literature}

Surprisingly enough given their importance in the applications, it seems that results on continuity and subdifferentiability for convex monotone maps have appeared only very recently in the literature.

After finishing the first version of the paper, which did not contain the subdifferentiability additional result, we came to know that in the recent articles [MMR] and [RS06] there are statements very close to those of Theorem 1.

To start, in $[\mathrm{MMR}]$ it is shown that:

if $L$ is an ordered Banach space, with $L_{+}$closed and such that $L=L_{+}-L_{+}$ then any convex monotone $\pi: U \rightarrow \mathbb{R}$ defined on an open set $U$ of $L$ is continuous.

These hypotheses are stronger than ours on the topological part as $L$ must be a Banach space, but milder on the order part. In fact, their conditions: the positive cone $L_{+}$is closed and generating $L=L_{+}-L_{+}$are always satisfied in a Frechet lattice. Note that nothing is said about subdifferentiability.

On the contrary, in [RS06] the authors were the first to prove subdifferentiability of convex monotone maps $\pi$, but with the stronger assumption that $\pi$ is defined on a Banach lattice $L$ : 
If $L$ is a Banach lattice, $\pi: L \rightarrow \overline{\mathbb{R}}$ is proper, convex and monotone, then it is continuous and subdifferentiable on the interior of the proper domain.

The line of their proof is the following. For any fixed $X^{*} \in \operatorname{int}(\operatorname{Dom}(\pi))$, first one exhibits a positive subgradient, which is then continuous by classic Namioka-Klee theorem. This implies lower semicontinuity of $\pi$ at $X^{*}$, which in turn implies continuity.

Inspired by this work we also prove subdifferentiability of $\pi$, in the case of Frechet lattices. However, we reverse the order, since first one proves continuity of $\pi$ on $\operatorname{int}(\operatorname{Dom}(\pi))$ and then subdifferentiability (this latter in the same way as done by [RS06]). This is only a matter of taste and it would not be difficult to extend the results in [RS06] with the same line of reasoning from Banach to Frechet lattices. The only interesting aspect in proving first continuity is that one realizes that the same proof of "classic" Namioka for linear positive functionals still holds, basically unchanged, for convex monotone maps.

The interested reader is also referred to [CL07] for further developments.

\section{On order lower semicontinuity in Riesz spaces}

Let us recall some basic facts about Riesz spaces. The same notation $\leq$ is used for the order relations in $\mathcal{R}$, in $(-\infty,+\infty]$ and for the direction of index sets of nets, as the meaning will be clear from the context.

A subset $A$ of a Riesz space $\mathcal{R}$ is order bounded if there exists $X_{1} \in \mathcal{R}$ and $X_{2} \in \mathcal{R}$ such that $X_{2} \leq X \leq X_{1}$ for all $X \in A$. A net $\left(X_{\alpha}\right)_{\alpha}$ in $\mathcal{R}$ is increasing, written $X_{\alpha} \uparrow$, if $\alpha \leq \beta$ implies $X_{\alpha} \leq X_{\beta}$. A net $\left(X_{\alpha}\right)_{\alpha}$ in $\mathcal{R}$ is increasing to some $X \in \mathcal{R}$, written $X_{\alpha} \uparrow X$, if $X_{\alpha} \uparrow$ and $\sup _{\alpha} X_{\alpha}=X$. A subset $A$ of $\mathcal{R}$ is order closed if $X_{\alpha} \in A$ and $X_{\alpha} \stackrel{o}{\rightarrow} X$ implies $X \in A$. The space $\mathcal{R}$ is order complete when each order bounded subset $A$ has a supremum (least upper bound) and an infimum (largest lower bound).

Recall ([ABo05, Th. 8.15]) that the lattice operations are order continuous. In addition ([ABo05, Th 8.16]), if a net $\left(X_{\alpha}\right)_{\alpha}$ is order bounded and $\mathcal{R}$ is order complete, then $\liminf _{\alpha} X_{\alpha} \triangleq \sup _{\alpha} \inf _{\beta \geq \alpha} X_{\beta}$ and $\lim \sup _{\alpha} X_{\alpha} \triangleq$ $\inf _{\alpha} \sup _{\beta \geq \alpha} X_{\beta}$ are well defined, and

$$
X_{\alpha} \stackrel{o}{\rightarrow} X \text { iff } X=\liminf _{\alpha} X_{\alpha}=\limsup _{\alpha} X_{\alpha}
$$

The next Lemma is an immediate consequence of the facts and definitions above and of (4).

Lemma 12 Let $\mathcal{R}$ be a Riesz space.

(i) Let $X_{\alpha} \stackrel{o}{\rightarrow} X$. Then there exists $\alpha^{*}$ such that $\left(X_{\alpha}\right)_{\alpha \geq \alpha^{*}}$ is order bounded, i.e. the net is definitely order bounded. In case the index set of the net has a minimum then $\left(X_{\alpha}\right)_{\alpha}$ is order bounded. 
(ii) Let $\mathcal{R}$ be order-complete and let $X_{\alpha} \stackrel{o}{\rightarrow} X$. If $Y_{\alpha} \triangleq\left(\inf _{\beta \geq \alpha} X_{\beta}\right) \wedge X$, then $Y_{\alpha} \uparrow X$.

Example 13 (Order convergence in $L^{p}$ ) In $L^{p}$ spaces, $p \in[0, \infty]$, the notion of order is the very familiar pointwise one, i.e. $Y \geq X$ iff $Y(\omega) \geq X(\omega)$ $P$-a.e. $A s L^{p}$ is order separable, see the next section, sequences can be used instead of nets to characterize order convergence. A sequence $\left(X_{n}\right)_{n}$ in $L^{p}$ is order bounded iff it is dominated in $L^{p}$ (i.e. there exists a $Y \in L_{+}^{p}$ such that $\left.\left|X_{n}\right| \leq Y\right)$. The order convergence in the $L^{p}$ case is just dominated pointwise convergence:

$$
X_{n} \stackrel{\circ}{\rightarrow} X \quad \Leftrightarrow \quad X_{n} \stackrel{P-a . e .}{\longrightarrow} X \quad \text { and }\left(X_{n}\right)_{n} \text { is dominated in } L^{p}
$$

Therefore, the $L^{p}$-norm topologies are order continuous for all $p<+\infty$, as the above equivalence implies that Lebesgue dominated convergence theorem can be applied to conclude $X_{n} \stackrel{o}{\rightarrow} X \Rightarrow X_{n} \stackrel{L^{p}}{\rightarrow} X$.

\subsection{Equivalent formulations of order l.s.c.}

Definition 14 A functional $\pi: \mathcal{R} \rightarrow(-\infty,+\infty]$ defined on a Riesz space $\mathcal{R}$

(a) is continuous from below if $X_{\alpha} \uparrow X \Rightarrow \pi\left(X_{\alpha}\right) \uparrow \pi(X)$

$\left(a_{\sigma}\right)$ is $\sigma$-continuous from below if $X_{n} \uparrow X \Rightarrow \pi\left(X_{n}\right) \uparrow \pi(X)$

(b) is order lower semicontinuous if $X_{\alpha} \stackrel{o}{\rightarrow} X \Rightarrow \pi(X) \leq \liminf \pi\left(X_{\alpha}\right)$

$\left(b_{\sigma}\right)$ is $\sigma$-order lower semicontinuous if

$$
X_{n} \stackrel{\circ}{\rightarrow} X \Rightarrow \pi(X) \leq \liminf \pi\left(X_{n}\right) .
$$

Note that the pointwise supremum of a family of order l.s.c. functionals is order l.s.c.

As shown in the next Lemma, if $\pi$ is increasing and $\mathcal{R}$ possesses more structure then the conditions $(a),\left(a_{\sigma}\right),(b),\left(b_{\sigma}\right)$ are all equivalent. The order separability of $\mathcal{R}$ (any subset $A$ which admits a supremum in $\mathcal{R}$ contains a countable subset with the same supremum) allows to formulate the order-l.s.c. property with sequences instead of nets (i.e. $(b) \Leftrightarrow\left(b_{\sigma}\right)$ ).

Lemma 15 Let $\mathcal{R}$ be an order complete Riesz space and $\pi: \mathcal{R} \rightarrow(-\infty,+\infty]$ be increasing. Then: $(a) \Leftrightarrow(b),\left(a_{\sigma}\right) \Leftrightarrow\left(b_{\sigma}\right),(a) \Rightarrow\left(a_{\sigma}\right),(b) \Rightarrow\left(b_{\sigma}\right)$.

If in addition $\mathcal{R}$ is order separable then $(a),\left(a_{\sigma}\right),(b),\left(b_{\sigma}\right)$ are all equivalent. 
Proof. $(a) \Rightarrow(b)$. Let $X_{\alpha} \stackrel{o}{\rightarrow} X$ and set $Y_{\alpha}=\left(\inf _{\beta \geq \alpha} X_{\beta}\right) \wedge X$. By Lemma 12 (ii), $Y_{\alpha} \uparrow X$ and so $\pi(X) \stackrel{(a)}{=} \lim \pi\left(Y_{\alpha}\right) \stackrel{(\text { mon })}{\leq} \liminf \pi\left(X_{\alpha}\right)$.

$$
(b) \Rightarrow(a) \text {. Since } X_{\alpha} \uparrow X \text { implies } X_{\alpha} \stackrel{o}{\rightarrow} X \text {, we get: } \pi(X) \stackrel{(b)}{\leq} \lim \pi\left(X_{\alpha}\right) \stackrel{(\text { mon })}{\leq}
$$
$\pi(X)$.

$\left(a_{\sigma}\right) \Leftrightarrow\left(b_{\sigma}\right)$ follows in the same way as $(a) \Leftrightarrow(b)$, while $(a) \Rightarrow\left(a_{\sigma}\right)$ and $(b) \Rightarrow\left(b_{\sigma}\right)$ are obvious.

To show the last sentence it is sufficient to prove e.g. $\left(a_{\sigma}\right) \Rightarrow(a)$. For any net $X_{\alpha} \uparrow X$ we can find a countable subnet $X_{\alpha_{n}}$ such that $X_{\alpha_{n}} \uparrow X$. Hence

$$
\pi(X) \stackrel{\left(a_{\sigma}\right)}{=} \lim _{n} \pi\left(X_{\alpha_{n}}\right) \leq \lim \pi\left(X_{\alpha}\right) \stackrel{(m o n)}{\leq} \pi(X) .
$$

Remark 16 (On order separability) A sufficient condition for $\mathcal{R}$ to be order separable is that, for every principal ideal $\mathcal{R}_{X}$, there exists a positive linear functional on $\mathcal{R}$ which is strictly positive on $\mathcal{R}_{X}$ (see [Za83, Theorem 84.4]).

All Banach lattices with order continuous norm verify this condition, as shown in [ABu85, Theorem 12.14].

Another sufficient condition for order separability is the existence of a linear functional on $\mathcal{R}$ which is strictly positive on the entire $\mathcal{R}$. This implies that all the Orlicz Banach lattices $L^{\Psi}=L^{\Psi}(\Omega, \mathcal{F}, P)$ (and henceforth all the $L^{p}$ spaces, $p \in[1, \infty]$ ) are order separable (and order complete as well). See Section 5.1.

Remark 17 (On the Fatou Property) From (9), Definition (2) and Definition (14), we immediate see that when $\mathcal{R}=L^{p}, p \in[0, \infty]$, order lower semicontinuity coincides with the Fatou Property.

Remark 18 (On decreasing functional) Analogous considerations hold for decreasing functionals: if $\mathcal{R}$ is an order complete and order separable Riesz space and if $\rho$ is decreasing, then the conditions: $(b),\left(b_{\sigma}\right)$, continuity from above [i.e.: $X_{\alpha} \downarrow X \Rightarrow \rho\left(X_{\alpha}\right) \uparrow \rho(X)$ ] and $\sigma$-continuity from above [i.e.: $\left.X_{n} \downarrow X \Rightarrow \rho\left(X_{n}\right) \uparrow \rho(X)\right]$ are all equivalent. These equivalent formulations will be used to study some properties of convex risk measures in Section 5.2.

\subsection{The order continuous dual $\mathcal{X}_{n}^{\sim}$}

Given a Frechet lattice $\mathcal{X}$, the space of order bounded linear functionals $\mathcal{X}^{\sim}$ (those which carry order bounded subset of $\mathcal{X}$ to order bounded sets of $\mathbb{R}$ ) coincides with the topological dual $\mathcal{X}^{\prime}$. This is a consequence of Namioka-Klee Theorem 7. From the general theory (see [Za83]) on the decomposition of $\mathcal{X}^{\sim}$

$$
\mathcal{X}^{\prime}=\mathcal{X}^{\sim}=\mathcal{X}_{n}^{\sim} \oplus \mathcal{X}_{s}^{\sim}
$$


where $\mathcal{X}_{n}^{\sim}$ is the order closed ideal (band) of $\mathcal{X}^{\sim}$ of all the order continuous linear functionals on $\mathcal{X}$ and it is called the order continuous dual of $\mathcal{X}$. The space of singular functionals $\mathcal{X}_{s}^{\sim}$ is defined as the band disjoint complement of $\mathcal{X}_{n}^{\sim}$ in $\mathcal{X}^{\sim}$. Examples of this decomposition are given in Section 5. The main goal of the next Section is to provide some criteria that guarantee the C-property of the topology $\sigma\left(\mathcal{X}, \mathcal{X}_{n}^{\sim}\right)$.

\section{On the C-property}

The C-property is verified by the strong topology of all Frechet lattices without passing to convex combinations, as shown below.

Lemma 19 Let $(\mathcal{X}, \tau)$ be a Frechet lattice. If $\left(X_{n}\right)_{n} \tau$-converges to $X$, then there exists a subsequence which is order convergent.

Proof. Call $d$ a complete distance that induces $\tau$, which is also absolute, i.e. $d(X, 0)=d(|X|, 0)$. Suppose $d\left(X_{n}, X\right) \rightarrow 0$ and select a subsequence such that $\sum_{k \geq 0} d\left(X_{n_{k}}, X\right)=\sum_{k \geq 0} d\left(X_{n_{k}}-X, 0\right)<+\infty$.

Set $Y=\sum_{k \geq 0}\left|X_{n_{k}}-X\right|$. By completeness of $d, Y \in \mathcal{X}$. Now, if $Y_{k}:=$ $\sum_{h \geq k}\left|X_{n_{h}}-X\right|$ then clearly $Y_{k} \downarrow$ and $Y_{k} \stackrel{\tau}{\rightarrow} 0$ so by [ABo05, Theorem 8.43] $Y_{k} \downarrow 0$. As

$$
\left|X_{n_{k}}-X\right| \leq Y_{k}
$$

one deduces that $X_{n_{k}}$ order converges to $X$.

We will be mainly concerned with the C-property of weak topologies in locally convex Frechet lattice. This is the reason why the most to hope for is to extract an order convergent subsequence of convex combinations from a topologically convergent net, e.g. exactly the C-property.

Lemma 20 Let $(\mathcal{X}, \tau)$ be a locally convex Frechet lattice. Then the $\sigma\left(\mathcal{X}, \mathcal{X}^{\prime}\right)$ topology verifies the C-property.

Proof. Let $W_{\alpha} \rightarrow W$ in the weak topology. By Hahn-Banach Theorem, $W$ belongs to the $\tau$-closure of $\operatorname{conv}\left(W_{\alpha}, \cdots\right)$ for all $\alpha$ and as the topology $\tau$ is first countable there exists a subsequence $\left(\alpha_{n}\right)_{n}$ and a sequence $Y_{n} \in \operatorname{conv}\left(W_{\alpha_{n}}, \ldots\right)$ which converges to $W$ in the $\tau$ topology. Lemma 19 ensures that we can extract a subsequence $\left(Y_{n_{k}}\right)_{k}$ that order converges to $W$.

Remark 21 The local convexity assumption cannot be dropped in the statement of the previous Lemma. An immediate counterexample is given by the Frechet lattice $L^{0}$, since when $P$ has no atoms $\left(L^{0}\right)^{\prime}=\{0\}$. So, the weak topology $\sigma\left(L^{0},\left(L^{0}\right)^{\prime}\right)$ is the indiscrete one and doesn't satisfy the C-property. 
However, even under the local convexity assumption, the C-property is not preserved if one keeps weakening the topology, from $\sigma\left(\mathcal{X}, \mathcal{X}^{\prime}\right)$ to $\sigma\left(\mathcal{X}, \mathcal{X}_{n}^{\sim}\right)$. An extreme situation is the one already encountered in Example 6 where the Banach lattice $\mathcal{X}=C([0,1])$ has dual $\mathcal{X}^{\prime}$ consisting of the signed measures $\mu$ of finite variation on $[0,1]$, but no $\mu$ is order continuous apart from the null measure.

So the following Lemma may be helpful.

Lemma 22 Let $\left(\mathcal{L}, \tau_{\mathcal{L}}\right),(\mathcal{X}, \tau)$ be locally convex Frechet lattices and suppose there exists a linear, injective lattice morphism

$$
(\mathcal{X}, \tau) \stackrel{i}{\rightarrow}\left(\mathcal{L}, \tau_{\mathcal{L}}\right)
$$

such that

$$
\left\{Y \circ i \mid Y \in \mathcal{L}^{\prime}\right\} \subseteq \mathcal{X}_{n}^{\sim} .
$$

Then $\sigma\left(\mathcal{X}, \mathcal{X}_{n}^{\sim}\right)$ verifies the C-property.

Proof. We specify that by "linear, injective lattice morphism" $i$ we mean that $i$ is linear, injective, topologically continuous and preserves the lattice structure. Note that $\mathcal{X}$ needs not to be homeomorphic to $i(\mathcal{X})$.

Let $\left(X_{\alpha}\right)_{\alpha}$ be a net such that $X_{\alpha} \stackrel{\sigma\left(\mathcal{X}, \mathcal{X}_{n}^{\sim}\right)}{\longrightarrow} X$. The condition (11) implies that $W_{\alpha}:=i\left(X_{\alpha}\right)$ converges to $W:=i(X)$ in the $\sigma\left(\mathcal{L}, \mathcal{L}^{\prime}\right)$-topology. Applying the same argument and using the same notations of the proof of Lemma 20, there exists $\left(Y_{n_{k}}\right)_{k}$ converging in order to $W$ in $\mathcal{L}$ and so the inverse image $Z_{k}=i^{-1}\left(Y_{n_{k}}\right)$ verifies $Z_{k} \in \operatorname{conv}\left(X_{\alpha_{n_{k}}}, \cdots\right)$ and $Z_{k} \stackrel{o}{\rightarrow} X$.

Condition (11) is evidently satisfied in case $\mathcal{L}^{\prime}=\mathcal{L}_{n}^{\sim}$, which is equivalent to the assumption that $\tau_{\mathcal{L}}$ is order continuous. If this holds, essentially the above Lemma applies to any locally convex Frechet lattice $\mathcal{X}$ that can be identified with a sublattice of $\mathcal{L}$, so the order structure is identical of that of $\mathcal{L}$, but with possibly finer topology than the one inherited from $\mathcal{L}$. This is the content of the next Corollary, that will be applied for the Orlicz Banach lattice $L^{\Psi}$.

Corollary 23 Any locally convex Frechet lattice $\mathcal{X}$ of random variables that can be injected into $L^{1}$ by a linear lattice morphism has $\tau, \sigma\left(\mathcal{X}, \mathcal{X}^{\prime}\right)$ and $\sigma\left(\mathcal{X}, \mathcal{X}_{n}^{\sim}\right)$ topologies with the C-property.

\subsection{The $C$-property in the representation of convex and monotone functionals}

We present the result on the equivalence between the $\sigma\left(\mathcal{X}, \mathcal{X}_{n}^{\sim}\right)-$ l.s.c. property for convex functionals on locally convex Frechet lattices and the order-l.s.c. property, under the assumption that the topology $\sigma\left(\mathcal{X}, \mathcal{X}_{n}^{\sim}\right)$ has the C-property. 
Proposition 24 Let $(\mathcal{X}, \tau)$ be a locally convex Frechet lattice and consider the following conditions for a proper, convex functional $\pi: \mathcal{X} \rightarrow(-\infty,+\infty]$ :

1. $\pi$ is $\sigma\left(\mathcal{X}, \mathcal{X}_{n}^{\sim}\right)-$ l.s.c.

2. $\pi$ admits the representation

$$
\pi(X)=\sup _{Y \in \mathcal{X}_{\tilde{n}}}\left\{\langle Y, X\rangle-\pi^{*}(Y)\right\}, \quad X \in \mathcal{X},
$$

3. $\pi$ is order l.s.c.

Then 1) $\Rightarrow 2) \Rightarrow 3$ ). If $\sigma\left(\mathcal{X}, \mathcal{X}_{n}^{\sim}\right)$ has the $C$-property, the three conditions are equivalent.

If $\pi$ is in addition monotone increasing, the conclusions are identical and in the representation $(12) \mathcal{X}_{n}^{\sim}$ can be replaced by $\left(\mathcal{X}_{n}^{\sim}\right)_{+}$.

Proof. 1) $\Rightarrow 2$ ) follows from $\left(\mathcal{X}, \sigma\left(\mathcal{X}, \mathcal{X}_{n}^{\sim}\right)\right)^{\prime}=\mathcal{X}_{n}^{\sim}$ and from Fenchel-Moreau Theorem (see e.g. [BR83, Chap I]);2) $\Rightarrow 3$ ) Since $\pi$ is the pointwise supremum of a family of order continuous functionals, it is also order l.s.c. Suppose now that $\sigma\left(\mathcal{X}, \mathcal{X}_{n}^{\sim}\right)$ has the $\mathrm{C}$-property and that 3 ) holds. To prove 1 ) we show that for any real $k$ the sublevel

$$
A_{k}=\{X \in \mathcal{X} \mid \pi(X) \leq k\}
$$

is $\sigma\left(\mathcal{X}, \mathcal{X}_{n}^{\sim}\right)$-closed. Suppose that $X_{\alpha} \in A_{k}$ and $X_{\alpha} \stackrel{\sigma\left(\mathcal{X}, \mathcal{X}_{n}^{\sim}\right)}{\rightarrow} X$. By the Cproperty, there exists $Y_{n} \in \operatorname{conv}\left(X_{\alpha_{n}}, \cdots\right)$ such that $Y_{n} \stackrel{o}{\rightarrow} X$. The convexity of $\pi$ implies that $\pi\left(Y_{n}\right) \leq k$ for each $n$. From order l.s.c. of $\pi$

$$
\pi(X) \leq \lim \inf \pi\left(Y_{n}\right) \leq k
$$

so that $X \in A_{k}$.

Remark 25 Note that the C-property could have been stated with order converging subnets of convex combinations instead of subsequences (as in fact it was in the first version of the present paper). However the current presentation is given with subsequences as the applications rely only on Corollary 23, which in turn is based on Lemma 20.

A natural question is whether the sup in formula (12) is attained when $\pi$ is finite valued. In general, the answer is no, as shown in the example below, where the max is attained over $\mathcal{X}_{+}^{\prime}$ thanks to (1) but not over $\left(\mathcal{X}_{n}^{\sim}\right)_{+}$.

Example 26 Consider the classic counterexample [FS04, Example 4.36] translated in the language of monotone increasing maps, that is take $\pi: L^{\infty} \rightarrow \mathbb{R}$, $\pi(X)=\operatorname{ess} \sup X$. This map is convex, increasing, positively homogeneous and 
order l.s.c. on $L^{\infty}$. For later use, observe also that it is not order u.s.c. From (12), taking into account the "cash additivity" property $\pi(X+c)=\pi(X)+c, c \in$ $\mathbb{R}$,

$$
\pi(X)=\sup _{\{Q \text { probab. }, Q \ll P\}} E_{Q}[X],
$$

Similarly, from (1) and the good properties of $\pi$,

$$
\pi(X)=\max _{Q \in \mathcal{M}_{1, f}(P)} E_{Q}[X]
$$

which is exactly the representation in (2) with zero penalty function. If $X$ is selected so that its ess-sup is not attained, the sup in (13) cannot be a maximum. We will consider a similar case in the Example 5.3.

If $\pi$ is finite valued and order u.s.c., then interestingly enough $\pi$ admits a representation as in (12) with the supremum replaced by a maximum, without the C-property requirement.

Lemma 27 Let $(\mathcal{X}, \tau)$ be a locally convex Frechet lattice and $\pi: \mathcal{X} \rightarrow \mathbb{R}$ be a convex increasing map. If $\pi$ is order u.s.c. then

$$
\pi(X)=\max _{Y \in\left(\mathcal{X}_{\tilde{n}}\right)_{+}}\left\{\langle Y, X\rangle-\pi^{*}(Y)\right\}, \quad X \in \mathcal{X},
$$

and thus a fortiori $\pi$ is order continuous.

Proof. From (1),

$$
\pi(X)=\max _{Y \in \mathcal{X}_{+}^{\prime}}\left\{\langle Y, X\rangle-\pi^{*}(Y)\right\} \geq \sup _{Y \in\left(\mathcal{X}_{\tilde{n}}\right)_{+}}\left\{\langle Y, X\rangle-\pi^{*}(Y)\right\}
$$

We now prove that any $Y$ attaining the max on $\mathcal{X}_{+}^{\prime}$ is order continuous. In fact, suppose by contradiction that the max is attained on a positive, non order continuous $Y_{0}$. Then, there exists $Z_{\alpha} \stackrel{o}{\rightarrow} 0$ such that $\lim \sup _{\alpha}\left\langle Y_{0}, Z_{\alpha}\right\rangle>0$ and

$\pi(X)=\left\{\left\langle Y_{0}, X\right\rangle-\pi^{*}\left(Y_{0}\right)\right\}<\lim \sup \left\{\left\langle Y_{0}, X+Z_{\alpha}\right\rangle-\pi^{*}\left(Y_{0}\right)\right\} \leq \lim \sup \pi\left(X+Z_{\alpha}\right)$ which is a contradiction with order u.s.c. of $\pi$.

\section{Orlicz spaces and applications to Risk Mea- sures}

\subsection{Orlicz spaces have the C-property}

The following Orlicz spaces and the $L^{p}$ spaces, $p \in[0,+\infty]$, are defined on the same probability space $(\Omega, \mathcal{F}, P)$.

A Young function $\Psi$ is an even, convex function $\Psi: \mathbb{R} \rightarrow \mathbb{R} \cup\{+\infty\}$ with the properties: 
1. $\Psi(0)=0$;

2. $\Psi(\infty)=+\infty$;

3. $\Psi<+\infty$ in a neighborhood of 0 .

Note that $\Psi$ may jump to $+\infty$ outside of a bounded neighborhood of 0 . In case $\Psi$ is finite valued however, it is also continuous by convexity.

The Orlicz space $L^{\Psi}$ is then defined as

$$
L^{\Psi}=\left\{X \in L^{0} \mid \exists \alpha>0 E[\Psi(\alpha X)]<+\infty\right\} .
$$

It is a Banach space with the Luxemburg (or gauge) norm

$$
N_{\Psi}(X)=\inf \left\{c>0 \mid E\left[\Psi\left(\frac{X}{c}\right)\right] \leq 1\right\} .
$$

With the usual pointwise lattice operations, $L^{\Psi}$ is also a Banach lattice, as the norm satisfies the monotonicity condition

$$
|Y| \leq|X| \Rightarrow N_{\Psi}(Y) \leq N_{\Psi}(X)
$$

Since $\Psi$ is bounded in a neighborhood of 0 and it is convex and goes to $+\infty$ when $|x| \rightarrow \infty$, it is rather easy to prove that

$$
L^{\infty} \stackrel{i}{\rightarrow} L^{\Psi} \stackrel{i}{\rightarrow} L^{1}
$$

with linear, injective lattice morphisms (the inclusions $i$ ). The dual $\left(L^{\Psi}\right)^{\prime}$ admits the general decomposition in order continuous band and singular band

$$
\left(L^{\Psi}\right)^{\prime}=\left(L^{\Psi}\right)_{n}^{\sim} \oplus\left(L^{\Psi}\right)_{s}^{\sim}
$$

and $\left(L^{\Psi}\right)_{n}^{\sim}$ can be identified with the Orlicz space $L^{\Psi^{*}}$ where

$$
\Psi^{*}(y)=\sup _{x \in \mathbb{R}}\{y x-\Psi(x)\}
$$

is the Young function conjugate of $\Psi$. The examples below illustrate different cases and show that the $L^{p}$ are in fact particular Orlicz spaces.

1. Suppose $p \in(1,+\infty)$ and $\Psi=\Psi_{p}$ where

$$
\Psi_{p}(x)=\frac{|x|^{p}}{p}
$$

then $L^{\Psi_{p}}=L^{p}$. Since this space has an order continuous topology, the dual consists only of order continuous functionals. As $\left(\Psi_{p}\right)^{*}=\Psi_{q}$ with $q=\frac{p}{p-1}$, one recovers the classic

$$
\left(L^{p}\right)^{\prime}=L^{q}
$$


2. $\Psi=\Psi_{\infty}$, where

$$
\Psi_{\infty}(x)=\left\{\begin{array}{cc}
0 & \text { if }|x| \leq 1 \\
+\infty & \text { otherwise }
\end{array}\right.
$$

Then the associated Orlicz space $L^{\Psi_{\infty}}$ is exactly $L^{\infty}$ and as $\left(\Psi_{\infty}\right)^{*}(y)=$ $|y|, L^{\left(\Psi_{\infty}\right)^{*}}=L^{1}$.

The decomposition of the dual provided in (15) is nothing but the YosidaHewitt decomposition $\left(L^{\infty}\right)^{\prime}=L^{1} \oplus\left(L^{\infty}\right)_{s}^{\sim}$ and the singular band $\left(L^{\infty}\right)_{s}^{\sim}$ consists of the purely finitely additive measures.

3. $\Psi_{e}(x)=e^{|x|}-1$ is a genuine example of Young function which induces an Orlicz space different from the $L^{p} . L^{\Psi_{e}}$ is the space of random variables with some finite exponential moment, i.e.

$$
L^{\Psi_{e}}=\left\{X \in L^{0} \mid \exists \alpha>0 \text { s.t. } E\left[e^{\alpha|X|}\right]<+\infty\right\} .
$$

Analogously to what happens for $L^{\infty}$, this space has a topology which is not order continuous. Thus the dual has the full general decomposition (15), with non-null singular band, as

$$
\left(L^{\Psi_{e}}\right)^{\prime}=L^{\left(\Psi_{e}\right)^{*}} \oplus\left(L^{\Psi_{e}}\right)_{s}^{\sim}
$$

where the conjugate $\left(\Psi_{e}\right)^{*}$ is given by the function

$$
\left\{\begin{array}{cc}
|y|(\ln |y|-1)+1 & \text { if }|y|>1 \\
0 & \text { otherwise }
\end{array}\right.
$$

which will be indicated with $\widehat{\Phi}$. As better explained below, since $\widehat{\Phi}$ doesn’t grow too fast the Orlicz $L^{\widehat{\Phi}}$ displays a behavior similar to that of the $L^{p}, 1 \leq p<+\infty$, in the sense that its topology is order continuous. Then, its dual $\left(L^{\widehat{\Phi}}\right)^{\prime}$ coincides with $L^{\widehat{\Phi}^{*}}=L^{\Psi_{e}}$. The consequence is that the topology induced on $L^{\Psi_{e}}$ by the order continuous functionals, $\sigma\left(L^{\Psi_{e}}, L^{\widehat{\Phi}}\right)$, is nothing but the weak ${ }^{*}$ topology on $L^{\Psi_{e}}$.

As anticipated in the examples above, when $\Psi$ verifies a slow- growth condition, known in the literature as $\Delta_{2}$ condition (see e.g. [RR91]):

$$
\exists t^{*}>0, \exists K>0 \text { s.t. } \Psi(2 t) \leq K \Psi(t) \text { for all } t>t^{*}
$$

then $\left(L^{\Psi}\right)^{\prime}=\left(L^{\Psi}\right)_{n}^{\sim}=L^{\Psi^{*}}$, that is the norm-topology on $L^{\Psi}$ is order continuous. So by Lemmata 19 and 20 the norm topology and the weak topology $\sigma\left(L^{\Psi},\left(L^{\Psi}\right)^{\prime}\right)=\sigma\left(L^{\Psi}, L^{\Psi^{*}}\right)$ have the C-property.

In general, by (14) and Corollary 23, the following topologies on $L^{\Psi}$ all have the C-property: (a) the norm topology, (b) the weak topology, (c) the $\sigma\left(L^{\Psi}, L^{\Psi^{*}}\right)$-topology. 
We remark that when it is $\Psi^{*}$ that satisfies the $\Delta_{2}$ condition, as in example 3 above, then the dual space of $L^{\Psi^{*}}$ coincides with $L^{\Psi}$. Therefore in this case the topology $\sigma\left(L^{\Psi}, L^{\Psi^{*}}\right)$ is nothing but the weak $k^{*}$ topology on $L^{\Psi}$ and it has the C-property.

One may also consider the Morse subspace $M^{\Psi}$ of the Orlicz space $L^{\Psi}$ :

$$
M^{\Psi}=\left\{X \in L^{\Psi} \mid E[\Psi(k X)]<+\infty \quad \forall k>0\right\} .
$$

When $\Psi$ is finite-valued, $M^{\Psi}$ is a norm closed band of $L^{\Psi}$ and its dual $\left(M^{\Psi}\right)^{\prime}=$ $L^{\Psi^{*}}$, so $\sigma\left(M^{\Psi}, L^{\Psi^{*}}\right)$ has the C-property too.

In the context of expected utility maximization, the spaces $M^{\Psi}$ were first used in [B07]. They are the object of study in [CL07] and applied to risk measures. In [CL07] it has also been shown that a risk measure defined on $M^{\Psi}$ has non empty topological interior if and only if it is finite valued. As the dual of the Morse space $M^{\Psi}$ can be identified with a space of functions, the Orlicz $L^{\Psi^{*}}$, these spaces are easier to handle than the whole $L^{\Psi}$. In particular, since $M^{\Psi}$ has order continuous norm, the dual representation (6) follows immediately from the Extended Namioka Klee Theorem 1.

In [BF08] and in [BFG07] it has been shown that the full duality $\left(L^{\widehat{u}},\left(L^{\widehat{u}}\right)^{\prime}\right)$ can also be successfully employed to cover new cases in the applications to expected utility maximization and indifference pricing. In fact the Orlicz space $L^{\widehat{u}}$, defined by the Young function $\widehat{u}(x)=-u(-|x|)+u(0)$ associated to the utility function $u$, is the natural environment for such investigation. And the results on the indifference price for claims in the general $L^{\widehat{u}}$ obtained in [BFG07] show that in the general setup the result by [CL07] fails: a convex risk measure on $L^{\widehat{u}}$ can have non empty topological interior without being finite valued everywhere. For other examples of this situation self-contained in the present paper, see the next Section 5.2 where there are some other interesting applications of the full duality to risk measures.

\subsection{New insights on the downside risk and risk measures associated to a utility function $u$}

We assume that the investment possibilities at a certain date in the future are modelled by elements $X$ of $L^{0}$. As straightforward consequences of Proposition 24 we have the following representations of decreasing functionals defined on subspaces of $L^{0}$.

Corollary 28 Let $(\mathcal{X}, \tau)$ be a locally convex Frechet lattice contained in $L^{0}$. If $\rho: \mathcal{X} \rightarrow(-\infty,+\infty]$ is a proper convex order l.s.c. decreasing functional and $\sigma\left(\mathcal{X}, \mathcal{X}_{n}^{\sim}\right)$ has the $C$-property, then $\rho$ admits the representation

$$
\rho(X)=\sup _{Y \in\left(\mathcal{X}_{n}^{\sim}\right)_{+}}\left\{\langle Y,-X\rangle-\rho^{*}(-Y)\right\}, \quad X \in \mathcal{X} .
$$


If in addition $\rho$ satisfies the cash additivity property

$$
\rho(X+c)=\rho(X)-c, \forall c \in \mathbb{R} \forall X \in \mathcal{X}
$$

then

$$
\rho(X)=\sup _{Y \in\left(\mathcal{X}_{\tilde{n}}\right)_{+},\langle Y, 1\rangle=1}\left\{\langle Y,-X\rangle-\rho^{*}(-Y)\right\}, \quad X \in \mathcal{X} .
$$

If in addition $\rho$ is positively homogeneous, then there exists a convex subset $\mathcal{C} \subseteq\left\{Y \in\left(\mathcal{X}_{n}^{\sim}\right)_{+} \mid\langle Y, 1\rangle=1\right\}$ such that

$$
\rho(X)=\sup _{Y \in \mathcal{C}}\langle Y,-X\rangle .
$$

Let us consider an agent, whose preferences on the investments $X$ can be represented via expected utility. We assume that the utility function

- $u: \mathbb{R} \rightarrow \mathbb{R}$ is increasing and concave (though not necessarily strictly concave) and satisfies $\lim _{x \rightarrow-\infty} u(x)=-\infty$.

Without loss of generality, suppose

$$
u(0)=0 .
$$

The goal is that of describing a natural framework associated to the expected utility of the agent, i.e. to the functional

$$
E[u(X)]
$$

to the related downside risk

$$
\Theta(X):=E[-u(X)]
$$

and to some associated convex risk measures. As it is not required that $u$ is strictly concave, $u$ can be identically 0 on $\mathbb{R}_{+}$and in this case $\Theta$ is nothing but the so-called shortfall risk ([FS04]).

It turns out that a good setup is that of an Orlicz spaces duality induced by the functional itself. As shown in [B07] and [BF08] the function

$$
\widehat{u}(x)=-u(-|x|)
$$

is a Young function and defines the Orlicz space $L^{\widehat{u}}$ associated to $u$. Call

$$
\Phi(y)=\sup _{x \in \mathbb{R}}\{u(x)-x y\}
$$

the convex conjugate of $u$. Since $\widehat{u}$ is finite on $\mathbb{R}$, then, as observed right after the definition (16), $M^{\widehat{u}}$ is a norm-closed band of $L^{\widehat{u}}$ and its dual is $L^{\widehat{\Phi}}$. 
It is clear that there must be a link between $\Phi$ and $\widehat{\Phi}$, the Young function conjugate to $\widehat{u}$. In fact

$$
\widehat{\Phi}(y)=\left\{\begin{array}{cc}
0 & \text { if }|y| \leq \beta \\
\Phi(|y|) & \text { if }|y|>\beta
\end{array}\right.
$$

where $\beta \geq 0$ is the right derivative of $\widehat{u}$ at 0 , namely $\beta=D^{+} \widehat{u}(0)=D^{-} u(0)$. If $u$ is differentiable, note that $\beta=u^{\prime}(0)$ and it is the unique solution of the equation $\Phi^{\prime}(y)=0$. To fix the ideas, consider the following examples.

1. Fix $\gamma>0$ and take

$$
u_{\gamma}(x)=-e^{-\gamma x}+1
$$

whence $\widehat{u_{\gamma}}(x)=e^{\gamma|x|}-1$ and

$$
\Phi_{\gamma}(y)=\frac{y}{\gamma} \ln \frac{y}{\gamma}-\frac{y}{\gamma}+1
$$

and $\widehat{\Phi}(y)=\left(\left|\frac{y}{\gamma}\right| \ln \left|\frac{y}{\gamma}\right|-\left|\frac{y}{\gamma}\right|+1\right) I_{\left\{\left|\frac{y}{\gamma}\right| \geq 1\right\}}$. It is not difficult to see that the associated Orlicz spaces do not depend on $\gamma$ (in the sense that they are physically the same and changing $\gamma$ amounts to a dilation of the Luxemburg norm) and therefore, as pointed out in Section 4.1 Example 3,

$$
\begin{gathered}
L^{\widehat{u_{\gamma}}}=\left\{X \in L^{0} \mid \exists \alpha>0 \text { s.t. } E\left[e^{\alpha|X|}\right]<+\infty\right\}, \\
M^{\widehat{u_{\gamma}}}=\left\{X \in L^{0} \mid \forall \alpha>0 \quad E\left[e^{\alpha|X|}\right]<+\infty\right\}, \\
L^{\widehat{\Phi_{\gamma}}}=\left\{Y \in L^{0} \mid E\left[(|Y| \ln |Y|) I_{\{|Y|>1\}}\right]<+\infty\right\} \text { and } \\
\left(L^{\widehat{u}}\right)^{\prime}=L^{\widehat{\Phi}} \oplus\left(L^{\widehat{u}}\right)_{s}^{\sim} .
\end{gathered}
$$

2. Let $u$ be the quadratic-flat utility, i.e.

$$
u(x)=\left\{\begin{array}{cc}
-\frac{x^{2}}{2} & \text { if } x \leq 0 \\
0 & \text { if } x \geq 0
\end{array}\right.
$$

In this case, $\widehat{u}(x)=\frac{x^{2}}{2}=\widehat{\Phi}(x)$, and all the spaces $L^{\widehat{u}}, M^{\widehat{u}}, L^{\widehat{\Phi}}$ are equal and coincide (modulo an isomorphism) with $L^{2}$.

Let us recall that the Orlicz class of $L^{\widehat{u}}$ is defined as

$$
\mathcal{L}^{\widehat{u}}=\left\{X \in L^{0} \mid E[\widehat{u}(X)]<+\infty\right\}
$$

and it is a convex subset (not necessarily closed) of $L^{\widehat{u}}$.

The following Lemma is a nice consequence of the right choice of the spaces. 
Lemma 29 The downside risk $\Theta: L^{\widehat{u}} \rightarrow(-\infty,+\infty], \Theta(X)=E[-u(X)]$, is a well-defined, proper, convex and monotone decreasing functional which is order l.s.c. In addition,

$$
\operatorname{Dom}(\Theta)=\left\{X \in L^{\widehat{u}} \mid X^{-} \in \mathcal{L}^{\widehat{u}}\right\}
$$

and

$$
\operatorname{int}(\operatorname{Dom}(\Theta))=\left\{X \in L^{\widehat{u}} \mid \exists \epsilon>0(1+\epsilon) X^{-} \in \mathcal{L}^{\widehat{u}}\right\} \supseteq M^{\widehat{u}} .
$$

Moreover, $\Theta$ admits the representation:

$$
\Theta(X)=\sup _{Y \in L_{+}^{\hat{\Phi}}}\{E[-X Y]-E[\Phi(Y)]\} .
$$

Proof. If $X \in L^{\widehat{u}}$, then by Jensen's inequality

$$
E[-u(X)] \geq-u(E[X])>-\infty
$$

since $E[X] \in \mathbb{R}$ from (14) and $u<+\infty$ on $\mathbb{R}$. So the definition is well-posed and $\Theta$ is clearly convex and monotone decreasing. To prove the characterization of $\operatorname{Dom}(\Theta)$, simply note that

$$
X \in \operatorname{Dom}(\Theta) \text { iff } E[u(X)]>-\infty \text { iff } E\left[u\left(-X^{-}\right)\right]>-\infty \text { iff } E\left[\widehat{u}\left(X^{-}\right)\right]<+\infty
$$

where the second equivalence above is due to the fact that $E\left[u\left(X^{+}\right)\right]$is always finite as

$$
u(0) \leq u\left(x^{+}\right) \leq a x^{+}+b
$$

for some $a, b \in \mathbb{R}$, so that $u(0) \leq E\left[u\left(X^{+}\right)\right] \leq a E\left[X^{+}\right]+b<+\infty$.

To prove (22), if $X \in \operatorname{int}(\operatorname{Dom}(\Theta))$ then clearly for some $\epsilon>0 E[-u(X-$ $\left.\left.\epsilon X^{-}\right)\right]$is finite, that is $E\left[\widehat{u}\left((1+\epsilon) X^{-}\right)\right]$is finite.

Conversely, suppose $(1+\epsilon) X^{-} \in \mathcal{L}^{\widehat{ }}$. Then, $(1+\epsilon) X \in \operatorname{Dom}(\Theta)$ and for any $Z$ with Luxemburg norm $N_{\widehat{u}}(Z)<\frac{\epsilon}{1+\epsilon}, X+Z \in \operatorname{Dom}(\Theta)$. In fact:

$$
\begin{aligned}
& E[-u(X+Z)]=E\left[-u\left(\frac{1}{1+\epsilon}((1+\epsilon) X)+Z\right)\right] \leq \\
& \frac{1}{1+\epsilon} E[-u((1+\epsilon) X)]+\frac{\epsilon}{1+\epsilon} E\left[-u\left(\frac{1+\epsilon}{\epsilon} Z\right)\right]<+\infty
\end{aligned}
$$

since $\frac{1+\epsilon}{\epsilon} Z$ has Luxemburg norm less than 1 and thus

$$
E\left[-u\left(\frac{1+\epsilon}{\epsilon} Z\right)\right] \leq E\left[-u\left(-\frac{1+\epsilon}{\epsilon} Z^{-}\right)\right]=E\left[\widehat{u}\left(\frac{1+\epsilon}{\epsilon} Z^{-}\right)\right] \leq 1
$$

Thanks to Remark 18, in order to show that $\Theta$ is order l.s.c. one just needs to check whether $\Theta$ is $\sigma$-continuous from above. But this is an immediate consequence of the monotone convergence theorem and (24). Finally, the $\sigma\left(L^{\widehat{u}}, L^{\widehat{\Phi}}\right)$ 
topology has the C-property so the representation (17) on the order continuous dual $L^{\widehat{\Phi}}$ applies

$$
\Theta(X)=\sup _{Y \in L_{+}^{\hat{\Phi}}}\left\{E[-Y X]-\Theta^{*}(-Y)\right\} .
$$

By Kozek's results [Koz79] (or directly by hand), $\Theta^{*}$, the convex conjugate of $\Theta$,

$$
\Theta^{*}(Y)=\sup _{X \in L^{\widehat{u}}}\{E[Y X]-\Theta(X)\}
$$

verifies

$$
\Theta^{*}(-Y)=E[\Phi(Y)], \text { if } Y \in L^{\widehat{\Phi}} .
$$

Clearly $\Theta$ satisfies all the requirements of a convex risk measure but cash additivity.

As shown in [BK06] in the $L^{\infty}$ case, the greatest convex risk measure smaller than a convex functional $\theta: L^{\infty} \rightarrow \mathbb{R}$ can be constructed by taking the infconvolution $\theta \square \rho_{\text {worst }}$ of $\theta$ with $\rho_{\text {worst }}=\rho_{L_{+}^{\infty}}$, which is the risk measure associated to the acceptance set $L_{+}^{\infty}$. Then the penalty function of $\rho_{\text {worst }}$ is equal to 0 on $\mathcal{M}_{1, f}(P)$, and is equal to $\infty$ outside $\mathcal{M}_{1, f}(P)$. Since the penalty function of $\theta \square \rho_{\text {worst }}$ is the sum of the penalty function of $\theta$ and of $\rho_{\text {worst }}$, the representation of $\theta \square \rho_{\text {worst }}$ will have the same penalty function of $\theta$, but the supremum in such representation is restricted to the set $\mathcal{M}_{1, f}(P)$, i.e. to those positive elements in the dual space that are also normalized. The same conclusion holds in our setting, as shown in the following result.

Proposition 30 The map $\zeta_{u}: L^{\widehat{u}} \rightarrow(-\infty,+\infty]$ defined by

$$
\zeta_{u}(X)=\sup _{Q \ll P, \frac{d Q}{d P} \in L_{+}^{\hat{\Phi}}}\left\{E_{Q}[-X]-E\left[\Phi\left(\frac{d Q}{d P}\right)\right]\right\}
$$

is a well-defined order l.s.c. convex risk measure and it is the greatest order l.s.c. convex risk measure smaller than $\Theta$ and hence $\zeta_{u}=\Theta \square \rho_{L_{+}^{\hat{u}}}$. Moreover, the sup in (26) can equivalently be computed on the set $\left\{Q\right.$ probab., $\left.Q \ll P \mid E\left[\Phi\left(\frac{d Q}{d P}\right)\right]<+\infty\right\}$.

Proof. It is clear that $\zeta_{u}$ is an order l.s.c convex risk measure. From (23) we also have: $\zeta_{u} \leq \Theta$. We need only to prove that if $\widetilde{\rho}: L^{\widehat{u}} \rightarrow(-\infty,+\infty]$ is an order l.s.c. convex risk measure such that $\widetilde{\rho} \leq \Theta$, then $\widetilde{\rho} \leq \zeta_{u}$. Let $\widetilde{\alpha}(Y)=\widetilde{\rho}^{*}(-Y)$ be the penalty function associated with $\widetilde{\rho}$ in the representation (19)

$$
\widetilde{\rho}(X)=\sup _{Y \in L_{+}^{\widehat{\Phi}}, E[Y]=1}\{E[-X Y]-\widetilde{\alpha}(Y)\} .
$$


By cash additivity, $\widetilde{\rho}(X+\Theta(X))=\widetilde{\rho}(X)-\Theta(X) \leq 0$, for all $X \in L^{\widehat{u}}$, so that

$$
\widetilde{\rho}(X+\Theta(X))=\sup _{Y \in L_{+}^{\hat{\Phi}}, E[Y]=1}\{E[-Y X]-\Theta(X)-\widetilde{\alpha}(Y)\} \leq 0 .
$$

This implies that, if $Y \in L_{+}^{\widehat{\Phi}}, E[Y]=1$,

$$
\widetilde{\alpha}(Y) \geq E[-Y X]-\Theta(X) \text { for all } X \in L^{\widehat{u}}
$$

and, by (25),

$$
\left.\widetilde{\alpha}(Y) \geq \sup _{X \in L^{\widehat{u}}}\{E[-Y X]-\Theta(X)]\right\}=\Theta^{*}(-Y)=E[\Phi(Y)] .
$$

Therefore,

$$
\widetilde{\rho}(X)=\sup _{Y \in L_{+}^{\hat{\Phi}}, E[Y]=1}\{E[-Y X]-\widetilde{\alpha}(Y)\} \leq \sup _{Y \in L_{+}^{\hat{\Phi}}, E[Y]=1}\left\{E[-Y X]-E[\Phi(Y)]=\zeta_{u}(X) .\right.
$$

Since the integrability condition $E[\Phi(Y)]<+\infty$ on $Y \geq 0$ is more severe than the requirement $Y \in L_{+}^{\widehat{\Phi}}$, the last sentence is obvious.

To any utility function satisfying our assumptions, one can also associate the map $\rho_{u}: L^{\widehat{u}} \rightarrow(-\infty,+\infty]$ defined by:

$$
\rho_{u}(X)=\inf \left\{c \in \mathbb{R} \mid X+c \in \mathcal{A}_{u}\right\},
$$

where the set $\mathcal{A}_{u}$ is defined as

$$
\mathcal{A}_{u}:=\left\{X \in L^{\widehat{u}} \mid E[u(X)] \geq u(0)=0\right\}=\left\{X \in L^{\widehat{u}} \mid \Theta(X) \leq 0\right\}
$$

Lemma $31 \mathcal{A}_{u}$ has the properties:

1. it is convex;

2. if $X \in \mathcal{A}_{u}$ and $Z \in L^{\widehat{u}}, Z \geq X$, then $Z \in \mathcal{A}_{u}$;

3. $\inf \left\{c \in \mathbb{R} \mid c \in \mathcal{A}_{u}\right\}>-\infty$;

4. for any $X \in \mathcal{A}_{u}$ and $Z \in L^{\widehat{u}}$, the set $\left\{t \in[0,1] \mid(1-t) X+t Z \in \mathcal{A}_{u}\right\}$ is closed in $[0,1]$.

Proof. We only prove item 4 , as the others are simple consequences of the properties of $u$. Fix any $X \in \mathcal{A}_{u}$ and call $\Lambda=\left\{t \in[0,1] \mid(1-t) X+t Z \in \mathcal{A}_{u}\right\}$. For any cluster point $t^{*}$ of $\Lambda$, there exists a sequence $\left(t_{n}\right)_{n} \in \Lambda, t_{n} \rightarrow t^{*}$. But then, $\left(1-t_{n}\right) X+t_{n} Z$ order converges to $\left(1-t^{*}\right) X+t^{*} Z$. From Lemma $29, \Theta$ is order l.s.c., so $\Theta\left(\left(1-t^{*}\right) X+t^{*} Z\right) \leq \liminf _{n} \Theta\left(\left(1-t_{n}\right) X+t_{n} Z\right) \leq 0$ which means $t^{*} \in \Lambda$. 
Proposition $32 \rho_{u}: L^{\widehat{u}} \rightarrow(-\infty,+\infty]$ is a well-defined order l.s.c. convex risk measure that admits the representation:

$$
\rho_{u}(X)=\sup _{Q \ll P, \frac{d Q}{d P} \in L_{+}^{\hat{\Phi}}}\left\{E_{Q}[-X]-\alpha(Q)\right\},
$$

where

$$
\alpha(Q)=\sup _{X \in \mathcal{A}_{u}}\left\{E_{Q}[-X]\right\} .
$$

Moreover, $\mathcal{A}_{\rho_{u}}:=\left\{X \in L^{\widehat{u}} \mid \rho_{u}(X) \leq 0\right\}$, the acceptance set of $\rho_{u}$, satisfies

$$
\mathcal{A}_{\rho_{u}}=\mathcal{A}_{u}
$$

and, as a consequence, if $\widetilde{\rho}: L^{\widehat{u}} \rightarrow \mathbb{R} \cup\{+\infty\}$ is an order l.s.c. convex risk measure such that $\widetilde{\rho} \leq \Theta$, then $\widetilde{\rho} \leq \rho_{u}$.

Proof. The facts that $\rho_{u}$ is a convex risk measure and that its acceptance set $\mathcal{A}_{\rho_{u}}$ coincides with $\mathcal{A}_{u}$ are consequences of the above Lemma and Propositions 2,4 in [FS02]. Now, since $\Theta$ is order l.s.c., $\mathcal{A}_{u}$ is order closed, so that the acceptance set of $\rho_{u}$ is order-closed. And since $\sigma\left(L^{\widehat{u}}, L^{\widehat{\Phi}}\right)$ has the C-property the acceptance set $\mathcal{A}_{\rho_{u}}=\mathcal{A}_{u}$ is $\sigma\left(L^{\widehat{u}}, L^{\widehat{\Phi}}\right)$-closed. Hence, by a classic result, as its sublevels are $\sigma\left(L^{\widehat{u}}, L^{\widehat{\Phi}}\right)$-closed, $\rho_{u}$ is $\sigma\left(L^{\widehat{u}}, L^{\widehat{\Phi}}\right)$-l.s.c. But this implies that it is also $\rho_{u}$ order l.s.c. by the first part of the statement in Proposition 24. Then, the representation (19) on the order continuous dual $L^{\widehat{\Phi}}$ applies:

$$
\begin{aligned}
& \rho_{u}(X)=\sup _{Q \ll P, \frac{d Q}{d P} \in L_{+}^{\widehat{\Phi}}}\left\{E_{Q}[-X]-\alpha(Q)\right\}, X \in L^{\widehat{u}}, \\
& \alpha(Q) \triangleq \rho_{u}^{*}(-Q)=\sup _{X \in L^{\widehat{u}}}\left\{E_{Q}[-X]-\rho_{u}(X)\right\}
\end{aligned}
$$

It is straightforward to see that the penalty functional $\alpha$ admits the representation

$$
\alpha(Q)=\sup _{X \in \mathcal{A} \rho_{u}} E_{Q}[-X]
$$

If $\widetilde{\rho}: L^{\widehat{u}} \rightarrow(-\infty,+\infty]$ is an order l.s.c. convex risk measure such that $\widetilde{\rho} \leq \Theta$ then $\mathcal{A}_{\widetilde{\rho}}=\left\{X \in L^{\widehat{u}} \mid \widetilde{\rho}(X) \leq 0\right\} \supseteq \mathcal{A}_{u}=\mathcal{A}_{\rho_{u}}$, and this implies $\widetilde{\rho} \leq \rho_{u}$.

Remark 33 Obviously, $\zeta_{u} \leq \rho_{u}$, but $\zeta_{u}=\rho_{u}$ if and only if $\rho_{u} \leq \Theta$ which in general is not true. Note that the inequality $\rho_{u} \leq \Theta$ would imply: $\Theta(X+$ $\Theta(X)) \leq \Theta\left(X+\rho_{u}(X)\right) \leq 0$ (this latter inequality follows from $X+\rho_{u}(X) \in$ $\left.\mathcal{A}_{\rho_{u}}=\mathcal{A}_{u}\right)$ ), but in general $\Theta(X+\Theta(X)) \leq 0$ does not hold. So, $\rho_{u}$ and $\zeta_{u}$ may be different (see the Subsection 5.3 below). In Subsection 5.4 there is a case where $\rho_{u}=\zeta_{u}$. 


\subsection{Quadratic-flat utility}

If $u$ is the quadratic-flat utility function (21), then $\zeta_{u}$ and $\rho_{u}$ are different. Indeed, $L^{\widehat{u}}=L^{2}$ and

$$
\begin{aligned}
\Theta(X) & =\frac{1}{2} E\left[\left(X^{-}\right)^{2}\right]=\sup _{Y \in L_{+}^{2}}\left\{E[-Y X]-\frac{1}{2} E\left[Y^{2}\right]\right\}, X \in L^{2}, \\
\zeta_{u}(X) & =\sup _{\frac{d Q}{d P} \in L_{+}^{2}}\left\{E_{Q}[-X]-\frac{1}{2} E\left[\left(\frac{d Q}{d P}\right)^{2}\right]\right\}, X \in L^{2} .
\end{aligned}
$$

Since $\mathcal{A}_{u}=\left\{X \in L^{2} \mid \Theta(X) \leq 0\right\}=L_{+}^{2}$, we have:

$$
\rho_{u}(X)=\inf \{c \in \mathbb{R} \mid X+c \geq 0\}=\rho_{\text {worst }}(X):=-\operatorname{essinf}(X) .
$$

The dual representation in (28) becomes

$$
\rho_{u}(X)=\sup _{Q \ll P, \frac{d Q}{d P} \in L_{+}^{2}} E_{Q}[-X]
$$

since, from (29), the penalty term is given by

$$
\alpha(Q)=\sup _{X \in L^{2}, \Theta(X) \leq 0} E_{Q}[-X]=0, \text { if } \frac{d Q}{d P} \in L_{+}^{2} .
$$

Note also that

$$
\rho_{u}(X)=\sup _{Q \ll P, \frac{d Q}{d P} \in L_{+}^{2}} E_{Q}[-X]=\sup _{Q \ll P, \frac{d Q}{d P} \in L_{+}^{2}} E_{Q}\left[X^{-}\right] \geq \frac{E\left[\left(X^{-}\right)^{2}\right]}{E\left[X^{-}\right]}
$$

and if $0<E\left[X^{-}\right]<2, \rho_{u}(X)>\Theta(X)$. Moreover, $\rho_{u}$ is not even finitevalued. Therefore, while $\Theta$ and $\zeta_{u}$ are finite valued and thus continuous and subdifferentiable on $L^{2}, \operatorname{Dom}\left(\rho_{u}\right)$ has empty interior thanks to the cited result of [CL07] for risk measures on Morse subspaces (here, $L^{\widehat{u}}=M^{\widehat{u}}=L^{2}$ ).

\subsection{Exponential utility}

Let $u(x)=-e^{-x}+1$ be the exponential utility function considered in (20). W.l.o.g. we set $\gamma=1$. Then,

$$
\Theta(X)=E\left[e^{-X}\right]-1, X \in L^{\widehat{u}},
$$

and $\Phi(y)=y \ln y-y+1$. From the definition (27) we have

$$
\left.\rho_{u}(X)=\inf \left\{c \in \mathbb{R} \mid E\left[e^{-X-c}-1\right] \leq 0\right]\right\}=\ln E\left[e^{-X}\right],
$$

with the convention $\ln E\left[e^{-X}\right]=+\infty$ if $E\left[e^{-X}\right]=+\infty$. Clearly $\rho_{u}(X)=$ $\ln E\left[e^{-X}\right] \leq E\left[e^{-X}\right]-1=\Theta(X)$ and therefore, in this case, $\rho_{u}=\zeta_{u}$. So, from 
(26) we recover the entropic risk measure together with its dual variational identity

$$
\begin{aligned}
\ln E\left[e^{-X}\right] & =\sup _{Q \ll P, \frac{d Q}{d P} \in L_{+}^{\widehat{\Phi}}}\left\{E_{Q}[-X]-E\left[\Phi\left(\frac{d Q}{d P}\right)\right]\right\} \\
& =\sup _{Q \ll P, \frac{d Q}{d P} \in L_{+}^{\hat{\Phi}}}\left\{E_{Q}[-X]-E_{Q}\left[\ln \left(\frac{d Q}{d P}\right)\right]\right\}, X \in L^{\widehat{u}} .
\end{aligned}
$$

The novelty here is that the space where this representation holds is $L^{\widehat{u}}$, naturally induced by $u$ and not an arbitrarily selected subspace of $L^{0}$ (traditionally, the entropic risk measure is defined on $L^{\infty}$ and the formula above is provided for $X \in L^{\infty} \subset L^{\widehat{u}}$, see [FS04] and the remarks below). And $\rho_{u}$ is a genuine example of a risk measure on the general Orlicz space $L^{\widehat{u}}$ which is not finite valued everywhere and still has domain with no empty interior: as $\operatorname{Dom}\left(\rho_{u}\right)=\operatorname{Dom}(\Theta)$, the interior of the domain has been computed in (22).

To conclude, let us focus on the restriction of $\rho_{u}=\zeta_{u}$ to the subspace $M^{\widehat{u}}$.

Corollary 34 The restriction $v_{u}$ of $\zeta_{u}$ to the subspace $M^{\widehat{u}}$ is a well-defined norm continuous (hence order continuous) convex risk measure $v_{u}: M^{\widehat{u}} \rightarrow \mathbb{R}$ that admits the representation

$$
v_{u}(X)=\max _{Q \ll P, \frac{d Q}{d P} \in L_{+}^{\hat{\Phi}}}\left\{E_{Q}[-X]-E\left[\Phi\left(\frac{d Q}{d P}\right)\right]\right\}, X \in M^{\widehat{u}} .
$$

We thus recover the representation formulae provided by [CL07] on Morse subspaces and the formula with the max for the entropic risk measure on $L^{\infty} \subset$ $M^{\widehat{u}}$.

\section{References}

[ABo05] C. D. Aliprantis and K. C. Border. Infinite dimensional analysis. Springer, 2005 (third edition).

[ABu85] C. D. Aliprantis and O. Burkinshaw. Positive Operators. Academic Press INC, 1985.

[ADEH99] Artzner P., F. Delbaen, J.M. Eber and D. Heath (1999): Coherent measures of risk, Mathematical Finance, 4, 203-228.

[BK06] P. Barrieu and N. El Karoui, Pricing, hedging and optimally designing derivatives via minimization of risk measures, To appear in Volume on Indifference Pricing (ed: Rene Carmona), Princeton University Press. 
[B07] S. Biagini. An Orlicz Spaces Duality for Utility Maximization in Incomplete Markets. Proceedings of Ascona 2005, Progress in Probability, Birkhäuser, 2007.

[BF08] S. Biagini and M. Frittelli. A Unified Framework for Utility Maximization Problems: an Orlicz Space Approach. Ann. Appl. Prob., Vol. 18/3, pp. 929-966, 2008.

[BFG07] S. Biagini, M. Frittelli and M. Grasselli. Indifference price for general semimartingales. Submitted, 2007.

[BR83] H. Brezis. Analyse fonctionnelle. Masson, 1983.

[CL07] P. Cheridito and T. Li. Risk Measures on Orlicz Hearts. Forthcoming on Math. Fin.

[De00] F. Delbaen. Coherent Risk Measures on General Probability Spaces. Essays in Honour of Dieter Sondermann. Springer Verlag, 2000.

[FS07] D. Filipovic and G. Svindland, The Canonical Model Space for LawInvariant Convex Risk Measures is $L^{\wedge} 1$, Preprint, 2008.

[FS04] H. Föllmer and A. Schied. Stochastic Finance. An Introduction in Discrete Time. De Gruyter, Berlin - New York, 2004 (second edition).

[FS02] Föllmer H. and A. Schied. Convex measures of risk and trading constraints. Finance \& Stochastics, Vol. 6, n. 4, 429-447, 2002.

[FR02] Frittelli M. and E. Rosazza Gianin. Putting order in risk measures, Journal of Banking \& Finance, Vol. 26, no. 7, 1473-1486, 2002.

[Koz79] A. Kozek. Convex integral functionals on Orlicz spaces. Annales Societatis Mathematicae Polonae, Series 1, Commentationes mathematicae XXI, 109-134., 1979.

[MMR] F. Maccheroni, M. Marinacci and A. Rustichini. A variational formula for the relative Gini concentration index. In press.

[Na57] I. Namioka. Partially ordered linear topological spaces. Mem. Amer. Math. Soc. no 24. Princeton University Press, 1957.

[RR91] M. M. Rao and Z. D. Ren. Theory of Orlicz Spaces. Marcel Dekker Inc., NY, 1991.

[RK08] L. Rüschendorf and M. Kaina, On convex risk measures on Lpspaces, preprint (2007). 
[RS06] A. Ruszczynski and A. Shapiro. Optimization of convex risk measures. Mat. Oper. Research, vol 31/3, 433-452, 2006.

[Za83] A. C. Zaanen. Riesz Spaces II. North Holland Math. Library, 1983. 